\title{
Optimum Disposition of Metal Particles in the Propellant Grain
}

\author{
Leonid L. Minkov, Ernst R. Shrager, and Elizaveta V. Pikushchak \\ Department of Physics and Engineering, Tomsk State University, 36 Lenin Avenue, Tomsk 634050, Russia \\ Correspondence should be addressed to Leonid L. Minkov; lminkov@ftf.tsu.ru
}

Received 19 August 2014; Accepted 23 October 2014; Published 12 November 2014

Academic Editor: Christopher J. Damaren

Copyright (C) 2014 Leonid L. Minkov et al. This is an open access article distributed under the Creative Commons Attribution License, which permits unrestricted use, distribution, and reproduction in any medium, provided the original work is properly cited.

\begin{abstract}
Using the dispersed metal in solid propellants to increase the temperature of combustion products leads to such a problem as the specific impulse loss due to the incomplete combustion of metal particles in the exhaust products. A redistribution of metal loaded into the propellant grain is one of the methods to decrease the specific impulse loss. This paper reports on the ways to obtain the optimum metal particle disposition for the case-bounded propellant grain of tube cross-sectional type. Three different approaches to analyze the metal combustion efficiency are discussed. The influence of the dynamic nonequilibrium of two-phase flow on the optimum metal particles disposition in the propellant grain of tube cross-sectional type is investigated.
\end{abstract}

\section{Introduction}

Increasing the energy efficiency of propulsion is an important task of designing solid rocket motors (SRM), one of the component parts of which is the creation of a propellant with the high specific impulse [1]. Composite solid propellants are currently widely used in SRM, which contain aluminium, magnesium, and other metals as fuel additives to increase the specific impulse and combustion stability [2].

It is known the metal additives, on the one hand, lead to improving the energy performance of composite solid propellant due to high temperature of combustion [3], and on the other hand, they are the sources of specific impulse losses due to incomplete combustion of metal particles (especially for small-scale SRM and nozzleless SRM) [4]. The latter can be decreased by the redistribution of metal particles inside a solid propellant in accordance with particularities of a propellant grain and SRM.

The problem of increasing the specific impulse due to the redistribution of metal inside the grain has been dealt with in the last 40 years. For example, in Japan in 1976 it was patented a composition of the solid propellant in which the metal redistribution is carried out by varying the particle size [5]. It was noted that, for the grain of SRM with the channel length to channel diameter ratio equal to 5-30, the redistribution of particle by sizes ( $44 \%$ of channel length is occupied by $50-150$ micron particles, $28 \%$ by $20-50$ micron particles, and $28 \%$ by less than 20 micron particles) can give a 4-7\% increase in the specific impulse as compared with uniform distribution of the metal powder inside of the propellant. In addition, the metal redistribution accomplished by changing its mass fraction is another way to increase the specific impulse.

A numerical investigation of the influence of the metal particle redistribution in accordance with a metal mass fraction on the combustion efficiency was carried out in paper [6] by assuming that the mass fraction of metal particles $z(X)$ varies linearly along the channel. It was shown that there exists an optimal slope of the line $z(X)$ providing maximum combustion efficiency for a given mass of metal in the propellant grain. The dependence of the coordinate of the point at which the metal particle leaves the grain surface on the initial size of the particle burning out at the channel outlet was given in the paper [7]. The solution was obtained for the flow in one-dimensional approximation and expressed in terms of modified Bessel functions.

Anyway, the study of the combustion efficiency of metal particles in the combustion chamber of SRM is based on the analysis of the motion of a single particle $[6,8]$. The motion of a single particle in the channel of solid propellant grain under the influence of various factors is well studied [9-13].

It is of interest to reveal the ways of an optimal disposition of metal particles in the propellant grain with a cylindrical 
channel from the maximum combustion efficiency point of view and to identify main parameters affecting the combustion efficiency.

\section{Zero-Dimensional Approach}

Combustion products of the metallized composite solid propellant (MCSP) leaving the burning surface are supposed to consist of metal $(\mathrm{Al})$, oxidizing gases $\left(\mathrm{CO}_{2}, \mathrm{H}_{2} \mathrm{O}\right.$, and $\left.\mathrm{O}\right)$, and inert gases (I). A chemical reaction takes place between the metal and the oxidizing gas to form a metal oxide and inert gases

$$
v_{m} \mathrm{Al}+v_{o} \mathrm{O}=v_{c} \mathrm{Al}_{2} \mathrm{O}_{3}+v_{i} \mathrm{I}
$$

Here $\mathrm{O}$ and $\mathrm{I}$ are the oxidizing gas and the inert gas, respectively. Equation (1) shows that complete combustion of one mole of metal requires $\nu_{o} / \nu_{m}$ moles of oxidizing gas. This stoichiometric ratio imposes certain restrictions on the permissible content of metal in the MCSP. We define the limit of the metal mass fraction in the MCSP $z_{\text {lim }}$, at which metal can burn completely. Number of gas moles generated at burning of the MCSP equals $\dot{G}_{\mathrm{bs}}(1-z) / M_{g}$. Number of metal moles admitted to the flow equals $\dot{G}_{\mathrm{bs}} z / M_{m}$. Therefore, in accordance with (1) for the complete combustion of metal the following equality must hold:

$$
\frac{\alpha \dot{G}_{\mathrm{bs}}(1-z) / M_{g}}{\dot{G}_{\mathrm{bs}} z / M_{m}}=\frac{v_{o}}{v_{m}} .
$$

Hence, the limit value of the mass fraction of metal can be found from

$$
z_{\lim }=\frac{1}{1+\omega / \alpha}
$$

Here $\omega=M_{g} \nu_{o} / M_{m} v_{m}$. Equation (3) shows that $z_{\lim }$ depends on $\alpha$ and the complex $\omega$. For values $z(\alpha)>z_{\lim }(\alpha)$ the metal cannot completely burn up, and for $z(\alpha)<$ $z_{\text {lim }}(\alpha)$ oxidizing gases will be sufficient to do it. For different propellants, the molar mass varies from 15 to $30 \mathrm{~kg} / \mathrm{Kmol}$, so that the complex $\omega$ varies from 0.8 to 1.6. It follows from (3) that decreasing the molecular weight of gas phase and increasing the mole fraction of oxidizing gas $\alpha$ lead to increasing the limit value of $z_{\text {lim }}(\alpha)$.

There are two main time parameters affecting the metal combustion completeness in the SRM, namely, the characteristic burning time of metal particle $t_{b}$ and the characteristic residence time of metal particle in the combustion chamber of the SRM, $t_{r}$.

In the case of zero-dimensional approach, the residence time can be defined as $t_{r}=\rho_{p} W_{\mathrm{cc}} / \dot{G}_{\text {out }}$. Assuming the nozzle discharge coefficient and the coefficient of thermal losses equal to unity, we find that

$$
t_{r}=\frac{W_{\mathrm{cc}}}{\Gamma(\gamma) A \sqrt{R T_{p}}},
$$

and here $\Gamma(\gamma)=\sqrt{\gamma}(2 /(\gamma+1))^{(\gamma+1) / 2(\gamma-1)}$.
The characteristic burning time of metal particle $t_{b}$ can be taken from the experimental dependence $[14,15]$. Here we use simpler correlation [15]:

$$
t_{b}=k \frac{D^{p}}{\alpha^{0.9}}
$$

where $k=1.062 \cdot 10^{4} \mathrm{sec} / \mathrm{m}^{1.5}$ for $p=1.5$. The condition of the complete combustion of metal one can be written in the form $\theta<1$, where $\theta=t_{b} / t_{r}$. When the ratio $W_{\mathrm{cc}} / A$ is $20 \mathrm{~m}$ and $\alpha$ is 0.1 , then the diameter of particles to be completely burned at the chamber outlet is less than $50 \mu \mathrm{m}$, and when $\alpha$ equals 0.4 , the diameter reaches $100 \mu \mathrm{m}$. Decreasing the volume of the combustion chamber leads to decreasing the diameter of particles which can completely burn. The estimate shows that when $W_{\text {cc }} / A=2 \mathrm{~m}$ and $\alpha=0.1$, the particle diameter reaches $10 \mu \mathrm{m}$ and when $\alpha=0.4$, the particle diameter $D$ is $25 \mu \mathrm{m}$.

Thus, the zero-dimensional approach allows us to derive conditions under which the metal particles in the combustion chamber of the SRM will be completely burned: (1) the mass fraction of metal particles in the MCSP has to be less than $z_{\text {lim }}$, determined from (3); (2) the ratio of the burning time of metal particle to the residence time of metal particle in the combustion chamber has to be less than unity, $\theta<1$.

\section{One- and Two-Dimensional Approaches}

The zero-dimensional approach does not allow us to detect the influence of particle distribution along the channel on the combustion completeness of particles. It can be made on the base of one- or two-dimensional approaches.

According to Larson [16], the metal combustion efficiency is defined in terms of mass flow rates as $E=1-\dot{G}_{m \text {,out }} / \dot{G}_{m \text {,bs }}$ and for the equilibrium flow (equality of gas and particles velocities), the main parameters affecting the efficiency are the ratio of the burning time of metal particle to the mean residence time of metal particle in the combustion chamber of the SRM, $\theta$, and the ratio of the mole fluxes of metal and oxidizer on the burning surface of propellant, $\Phi$, taking into account the stoichiometric ratio $\nu_{o} / \nu_{m}$, which can be written as

$$
\Phi=\frac{v_{o}}{v_{m}} \frac{\rho_{m} w_{m} / M_{m}}{\alpha_{0} \rho_{g} w_{g} / M_{g}}, \quad \theta=\frac{2 w_{m}}{R_{k}} \frac{k D_{0}^{p}}{\alpha_{0}^{0.9}}
$$

At $w_{g}=w_{s}=w$, we have

$$
\Phi=\frac{z}{1-z} \frac{\omega}{\alpha_{0}} \quad \theta=\frac{2 w}{R_{k}} \frac{k D_{0}^{p}}{\alpha_{0}^{0.9}} .
$$

The parameter $\Phi$ is greater unity in the case of the oxidizer deficiency and $\Phi$ is less unity in the case of the oxidizer excess.

As a rule, the MCSP consists of three main components: a binder, an oxidizer, and powdered metal. A mass fraction of metal distributed in the propellant can be expressed through mass densities and volume fractions of the components from

$$
\frac{z}{1-z}=\frac{\beta_{\mathrm{mb}} \rho_{m}}{\left(1-\beta_{\mathrm{mb}}\right) \rho_{b}+\left(\beta_{\mathrm{ox}} /\left(1-\beta_{\mathrm{ox}}\right)\right) \rho_{\mathrm{ox}}},
$$


where $\beta_{\mathrm{mb}}$ is the volume fraction of metal distributed in the binder, equal to $\beta_{m} /\left(\beta_{\mathrm{bi}}+\beta_{m}\right)$. Since the velocity of combustion products leaving the burning surface of the SRM is expressed in terms of the temperature through the correlation

$$
w=\frac{A}{S_{\mathrm{bs}}} \sqrt{R T_{p}} \Gamma(\gamma),
$$

the formula for the parameter $\theta$ is

$$
\theta=\frac{2 A}{S_{\mathrm{bs}}} \frac{\sqrt{R T_{p}} \Gamma(\gamma)}{R_{k}} \frac{k D_{0}^{p}}{\alpha_{0}^{0.9}} .
$$

The necessary conditions for the complete combustion of metal in the propellant grain are $\Phi<1$ and $\theta<1$. Following (3), (7) the case $\Phi>1(\Phi<1)$ corresponds to $z>z_{\lim }(z<$ $\left.z_{\lim }\right)$.

Analysis of (7)-(10) shows that the increase of the mass fraction of metal leads to the increase in the parameter $\Phi$ and the decrease in the parameter $\theta$ and the increase of the mole fraction of oxidizer $\alpha_{0}$ decreases both $\Phi$ and $\theta$. To evaluate parameters $\Phi$ and $\theta$, letting us take $A / S_{\mathrm{bs}}=1.12 \cdot 10^{-2}, T_{p}=$ $3500^{\circ} \mathrm{K}, z=0.2, \alpha_{0}=0.4, \gamma=1.2, M_{g}=24 \mathrm{~kg} / \mathrm{Kmol}$, and $R=285 \mathrm{~J} /(\mathrm{kg} \cdot \mathrm{K})$, we have $\Phi=0.8333, \theta=0.366$ for $D_{0}=$ $50 \mu \mathrm{m}$, and $\theta=1.038$ for $D_{0}=100 \mu \mathrm{m}$.

The residence time of combustion products in the chamber of SRM defined by (4) and used in (10) is an average characteristic, so even if $\theta<1$, it does not mean that all the metal from the burning surface of channel is completely burned. Indeed, the metal particle leaving the area located closer to the front end of grain requires more time for the motion before the exiting channel than the particles leaving the area located near the exit of channel. If $\Phi<1$, there exists a coordinate $X_{\lim }$ of a point at the burning propellant surface from which the particle escapes and burns out at the exit of channel. Therefore, at $X<X_{\lim }$ particles burn completely out before the exit, whereas at $X>X_{\lim }$ they do not.

To estimate the coordinate $X_{\lim }$ and to define the residence time of metal particle $t_{d}$, one can use the approximation of the equilibrium two-phase flow. The rotational flow field in a channel of constant cross-section with a lateral burning surface is given as follows [17]:

$$
\begin{aligned}
& U_{g}=\pi \frac{X}{R_{k}} w \cdot \cos \left[\frac{\pi}{2}\left(\frac{Y}{R_{k}}\right)^{2}\right] ; \\
& V_{g}=-\frac{R_{k}}{Y} w \cdot \sin \left[\frac{\pi}{2}\left(\frac{Y}{R_{k}}\right)^{2}\right]
\end{aligned}
$$

and the irrotational one is given as follows [18]:

$$
U_{g}=2 \frac{X}{R_{k}} w ; \quad V_{g}=-\frac{Y}{R_{k}} w .
$$

In the case of one-dimensional approach, the continuity equation gives the velocity of product combustion in the form $U=2 X w / R_{k}$, which coincides with the velocity of the irrotational flow field for two-dimensional case.
One can see from the above correlations that the axial velocity in the case of rotational flow field depends on both $X$ and $Y$ coordinates and in the other cases it depends only on $X$ coordinate. Therefore, to find a dependent $X(t)$ for the metal particle in the case of the rotational flow field, we have to solve the system of two equations $X / d t=U_{g} ; d Y / d t=V_{g}$; $X(0)=X_{0} ; Y(0)=R_{k}$ and one equation $d X / d t=U_{g} ; X(0)=$ $X_{0}$ otherwise. Solving the equations gives for the rotational flow field:

$$
X=X_{0} \cosh \left(\frac{\pi w}{R_{k}} t\right)
$$

and for the irrotational flow field

$$
X=X_{0} \exp \left(\frac{2 w}{R_{k}} t\right) .
$$

From formulas (13) and (14) it follows that if the particle burning time equals the average residence time of the particle in the channel, $\theta=1, t_{b}=R_{k} /(2 w)$, the value of $X_{\text {lim }} / L$ equals 0.398 for the rotational flow and 0.367 for irrotational flow. It means that particles from more than half of the propellant grain surface do not burn out.

In the limiting case when $\Phi \rightarrow 0$ and $p=1.5$, the metal combustion efficiency is yielded by the following.

For one-dimensional flow,

$$
E(\theta)=\frac{2}{\theta}\left(1+\frac{\exp (-\theta)-1}{\theta}\right) .
$$

For two-dimensional flow,

$$
E(\theta)=2 \int_{0}^{1} \frac{1-\zeta}{\cosh (\pi \theta \zeta / 2)} d \zeta
$$

So for the uniform distribution of particles along the channel, the value of the metal combustion efficiency is less unity, $E(1)=0.686$ and $E(1)=0.605$ for two-dimensional and one-dimensional cases, respectively.

\section{Results and Discussions}

4.1. Optimum Distribution along the Grain. To provide the equality of the metal combustion efficiency to unity, it is necessary that the residence time of the particle from the point with $X_{0}$-coordinate should be greater than its burning time. One can see from the expression (10) for $\theta$ that this may be achieved by variation of $D_{0}$ or $w$ at constant values of $R_{k}$ and $\alpha_{0}$. Let us define the dependent $\theta(X)$ giving the value of $E$ equal to unity.

As far as the gas is assumed to be incompressible and flow field is equilibrium, the mole fraction of oxidizer along the particle trajectory can be written as [16]

$$
\alpha=\alpha_{0}+\frac{\rho_{m}}{\rho_{g}} \omega\left[\left(\frac{D}{D_{0}}\right)^{3}-1\right]
$$

which is valid for both one-dimensional and two-dimensional approaches. The burn rate law for the particle can be represented as

$$
\frac{d D}{d t}=-\frac{1}{k p} D^{1-p} \alpha^{0.9}
$$


Passing to the dimensionless variables $\delta=D / D_{0}, \tau=t 2 w / R_{k}$ gives

$$
\frac{d \delta}{d \tau}=-\frac{1}{p \theta} \delta^{1-p}\left[1+\Phi\left(\delta^{3}-1\right)\right]^{0.9}
$$

Denoting $f(\delta, \Phi)=\delta^{p-1}\left[1+\Phi\left(\delta^{3}-1\right)\right]^{-0.9}$ and integrating (19) by the time from 0 to the burning time of particle $\tau_{b}$, we obtain

$$
\tau_{b}=p \theta \int_{0}^{1} f(\delta, \Phi) d \delta .
$$

Using previously obtained relations (13), (14) and introducing the variable $\xi=X_{0} / L$, we have for two-dimensional flow

$$
\theta(\xi)=\frac{(2 / \pi p) \operatorname{arcosh}(1 / \xi)}{\int_{0}^{1} f(\delta, \Phi) d \delta}
$$

and for one-dimensional flow

$$
\theta(\xi)=\frac{(1 / p) \ln (1 / \xi)}{\int_{0}^{1} f(\delta, \Phi) d \delta}
$$

Thus, it follows from (21), (22), which can be considered as the optimal distribution of the metal particles, that $E=1$ can be reached by a variation of $\theta$ along $\xi$ at $\Phi=$ const and by a variation of $\Phi$ along $\xi$ at $\theta=$ const. Assuming $\Phi=0$, we have the following.

For two-dimensional flow,

$$
\theta(\xi)=\frac{2}{\pi} \operatorname{arcosh}\left(\frac{1}{\xi}\right) .
$$

For one-dimensional flow,

$$
\theta(\xi)=\frac{1}{p} \ln \left(\frac{1}{\xi}\right) .
$$

At $\Phi \rightarrow 1$ in both cases, we have $\theta(\xi) \rightarrow \delta(\xi)$-Dirac delta function. Meaning of the latter is that when $\Phi=1$, the value of parameter $\theta$ (and hence the particle size) should be zero along the entire channel, except for the point $\xi=0$.

Figure 1 shows characteristic profiles of $\theta(\xi)$ at different values of the parameter $\Phi$. The dashed curves correspond to the one-dimensional flow and the solid ones to the twodimensional flow. Qualitative difference can be clearly seen in the behavior of functions $\theta(\xi)$ when $\xi \rightarrow 1$ and for both cases $\theta \rightarrow \infty$ when $\xi \rightarrow 0$.

Behavior of $\Phi$ along the length of the channel at different values of the parameter $\theta$ is depicted in Figure 2. It is seen that to achieve the equality $E=1$ the metal particles should be absent in the propellant to the right of the intersection point between the abscissa axis and the curve $\Phi(\xi)$. The increase (decrease) of the value of $\theta$ leads the shift of the intersection point to the left (right) and for one-dimensional flow (dashed curves) the region free of particles are greater than those for two-dimensional flow (solid curves).

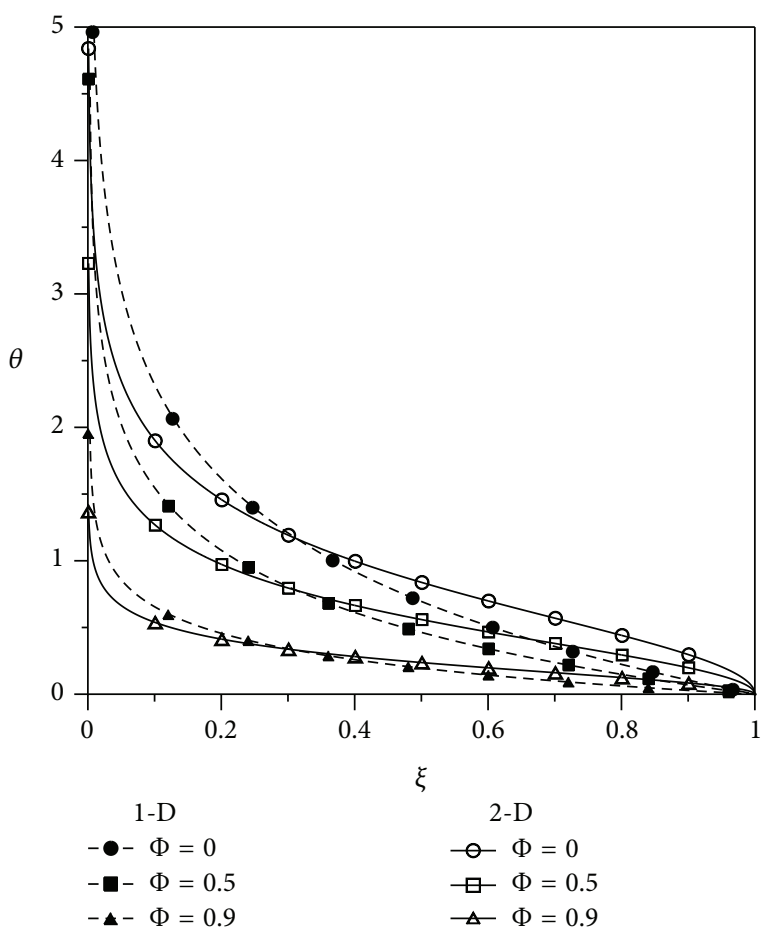

Figure 1: Parameter $\theta$ distribution along the channel.

4.2. Optimum Distribution across the Grain. During the propellant combustion process, the channel radius decreases that leads to the change of the value of $\theta$ for a given value of $\xi$. Assuming that the increase of the combustion chamber volume does not affect the temperature in the combustion chamber, the value of $\theta$ is inversely proportional to the channel radius squared in the case of the SRM with the geometry of $A / S_{\mathrm{bs}} \sim 1 / R_{k}$ as follows from (10). The inverse proportionality to the channel radius takes place for SRM with the geometry of $A / S_{\mathrm{bs}}=$ const. Therefore, when the value of $R_{k}$ increases, the value of $\theta$ decreases, and dependent $\Phi(\xi)$ and $\theta(\xi)$ will change. From (21) and (22), one can obtain next correlation

$$
p \theta \int_{0}^{1} f(\delta, \Phi) d \delta=g(\eta) h(\xi)
$$

where

$$
\begin{aligned}
h(\xi)= \begin{cases}\frac{2}{\pi} \operatorname{arcosh}\left(\frac{1}{\xi}\right), & 2 \text {-D flow } \\
\ln \left(\frac{1}{\xi}\right), & \text { 1-D flow; }\end{cases} \\
g(\eta)= \begin{cases}\eta, & \frac{A}{S_{\mathrm{bs}}}=\text { const } \\
\eta^{2}, & \frac{A}{S_{\mathrm{bs}}} \sim \frac{1}{R_{k}},\end{cases}
\end{aligned}
$$

where $\eta$ is the ratio of the current radius of the channel to the initial one. The correlation (25) should be treated either as a relation between the parameters $\Phi$ and $\theta$ at constant value of its RHS, which provides $E=1$, or as an equation for determining the function $\theta(\xi, \eta)$ at given $\Phi$ (position 


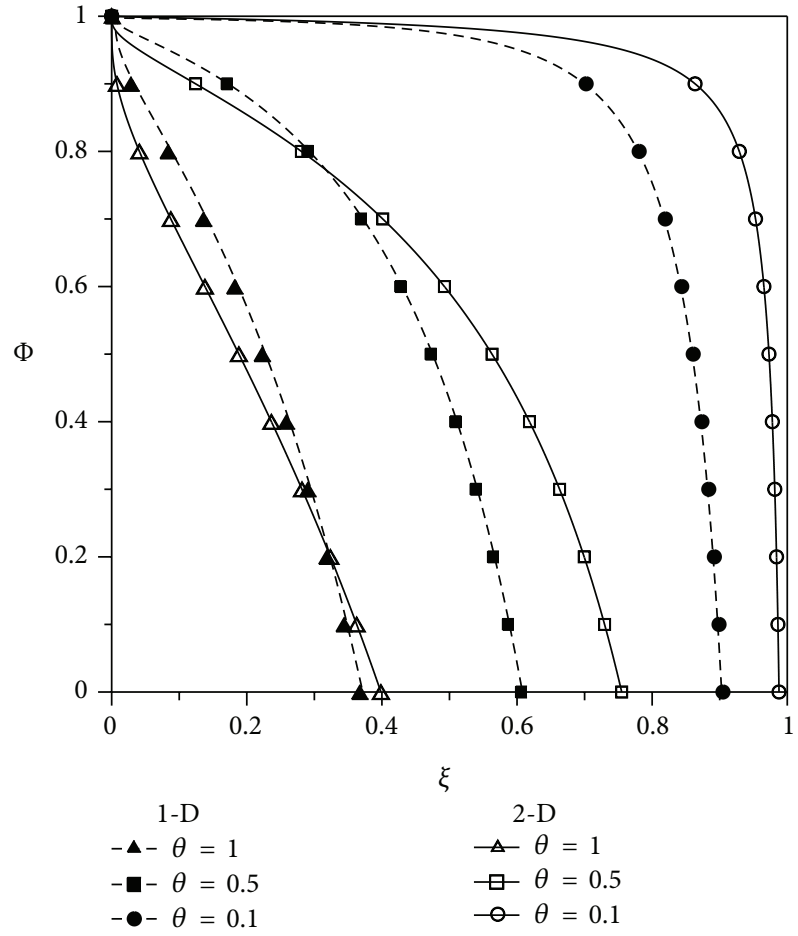

Figure 2: Parameter $\Phi$ distribution along the channel.

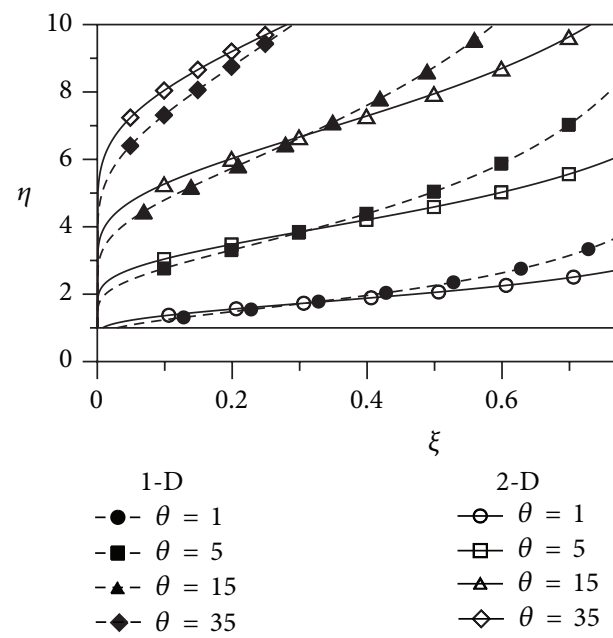

FIgURE 3: Optimum profiles of metal content at $\Phi=0.9, g(\eta)=\eta^{2}$.

of the equal size particles inside the propellant which can completely burn at the channel outlet), or as an equation for determining the function $\Phi(\xi, \eta)$ at given $\theta$ (position of propellant portions with the equal mass fraction of metal providing the complete combustion of particles).

Profiles of $\eta(\xi)$ for 2D flow (solid curves) and for 1D flow (dashed curves) are obtained at $\Phi=0.99, g(\eta)=\eta^{2}$ and different values of $\theta$ are depicted in Figure 3. Curves corresponding to different kinds of flow are close to each other near the front end of the grain and are quite different near the rear end of the grain. The distribution of equal size particles $(\theta=1)$ in accordance with their mass content inside the propellant grain is shown in Figure 4.

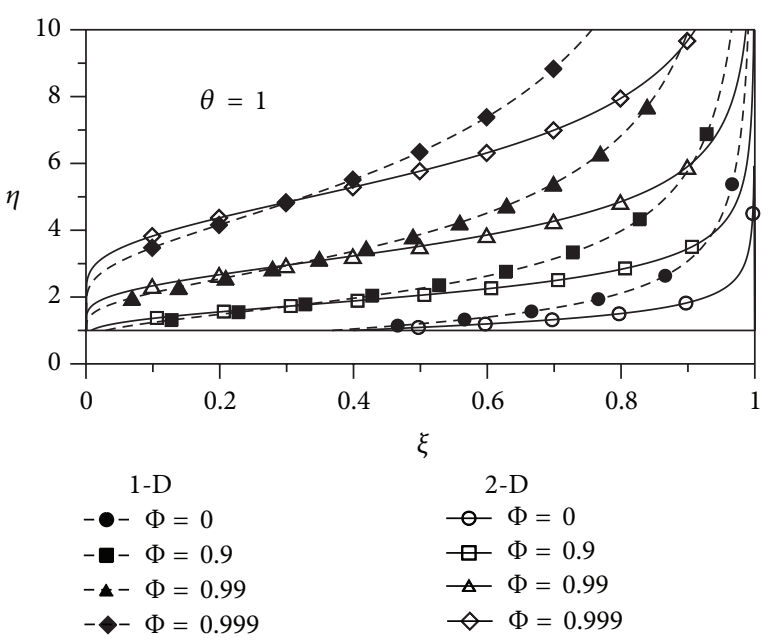

FIGURE 4: Optimum profiles of metal content at $\theta=1, g(\eta)=\eta^{2}$.

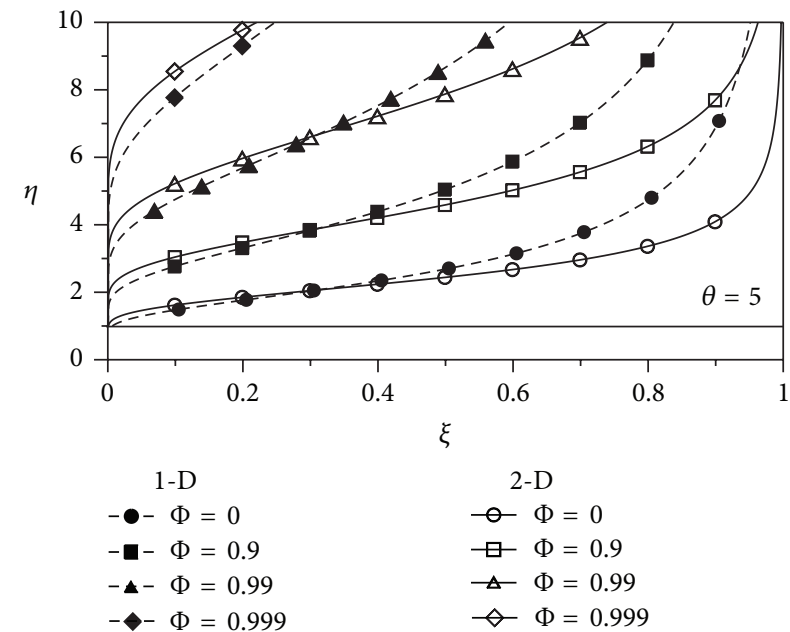

FIGURE 5: Optimum profiles of metal content at $\theta=5, g(\eta)=\eta^{2}$.

The increase of $\theta$ (Figure 5) leads to enhancing the region where $\Phi$ changes from 0 to 0.9 and increasing a zone free of particles (part of the grain below the curve $\Phi=0$ ). The same behaviour of $\eta(\xi)$ takes place for $g(\eta)=\eta$ and $\theta=1$ (Figure 6). The fact that the particle residence time in the case of the $1 \mathrm{D}$ flow is less than that that in the case of the $2 \mathrm{D}$ flow leads to a displacement of profiles $\theta=$ const (Figure 4) and $\Phi=$ const (Figure 6) downwards at the front end of the grain and upwards at the rear end of the grain with respect to the similar profiles for $2 \mathrm{D}$ flow.

Figure 7 shows the dependence of the parameter $\theta$ to complex $g(\eta) h(\xi)$ ratio on the parameter $\Phi$ at $g(\eta) h(\xi)=$ const. It is seen that at $\Phi=0$ (the flux of metal is negligible in comparison with the flux of oxidizing gas) the parameter $\theta$ can be arbitrary due to the arbitrariness of values of $g(\eta) h(\xi)$ and at $\Phi=1$ (stoichiometric ratio of mole fluxes) the value of $\theta$ should be equal to zero.

4.3. Effect of the Velocity Lag. The moving inertial particle has a velocity less than the velocity of the gas whereby 


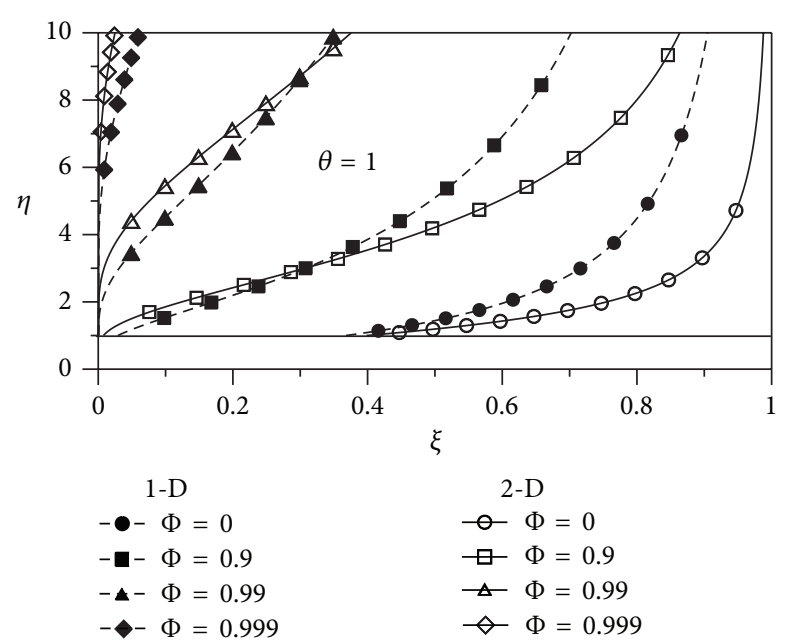

FIGURE 6: Optimum profiles of metal content at $\theta=1, g(\eta)=\eta$.

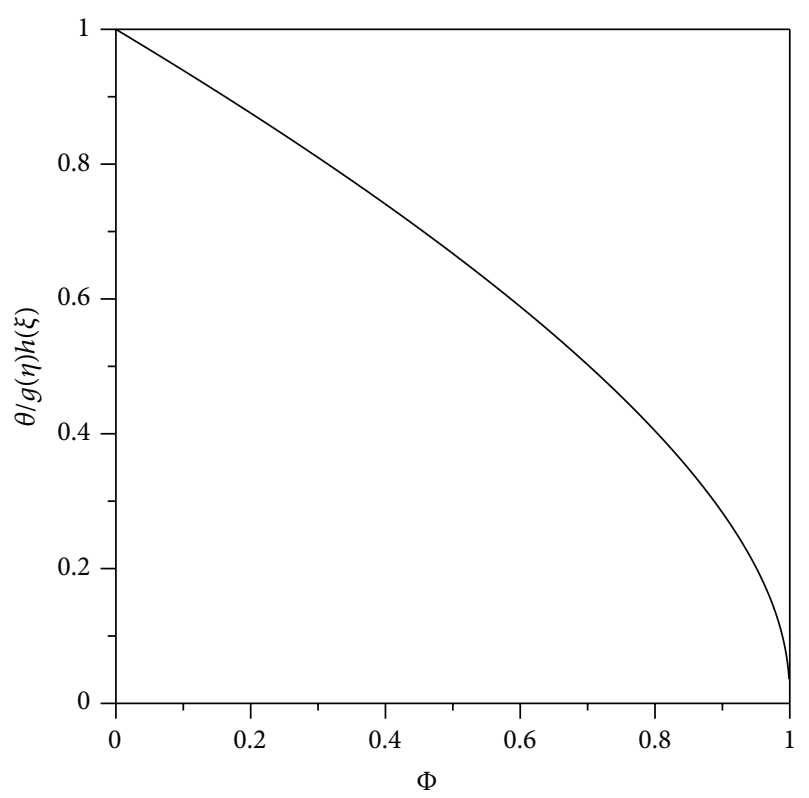

FIGURE 7: Relationship between parameters of $\Phi$ and $\theta$.

the residence time of the particles in the channel increases, which increases the metal combustion efficiency.

Consider the two-dimensional approximation. Following [16], we can write the system of equations describing the motion of a particle in the channel flow field taking into account the fact that the particle velocity lag is negligible at the calculation of the oxidizer concentration along the particle trajectory (so-called King's model):

$$
\begin{gathered}
\frac{d \delta}{d \tau}=-\frac{1}{p \theta} \delta^{1-p}\left[1+\Phi\left(\delta^{3}-1\right)\right]^{0.9} \\
\frac{d u_{m}}{d \tau}=\frac{9 \lambda}{\psi \delta^{2}}\left(u_{g}-u_{m}\right) ; \quad \frac{d v_{m}}{d \tau}=\frac{9 \lambda}{\psi \delta^{2}}\left(v_{g}-v_{m}\right) \\
\frac{d x}{d \tau}=u_{m} ; \quad \frac{d y}{d \tau}=v_{m}
\end{gathered}
$$

where

$$
\begin{gathered}
\lambda=1+0.15 \operatorname{Re}_{1}^{0.687} ; \\
\operatorname{Re}_{1}=2 \delta \operatorname{Re} \sqrt{\left(u_{g}-u_{m}\right)^{2}+\left(v_{g}-v_{m}\right)^{2}} \\
\operatorname{Re}=\frac{D_{0} \rho_{g} w}{\left(1-\beta_{m}\right) \mu_{g}} ; \quad \psi=\frac{\rho_{m} D_{0}^{2} w}{\beta_{m} \mu_{g} R_{k}} .
\end{gathered}
$$

The system of (27) is dimensionless, where the length scale is chosen to be the radius of the channel and the velocity scale is chosen to be $2 w$.

For rotational flow,

$$
u_{g}=\frac{\pi}{2} x \cdot \cos \left(\frac{\pi}{2} y^{2}\right) ; \quad v_{g}=-\frac{1}{2 y} \sin \left(\frac{\pi}{2} y^{2}\right),
$$

and for irrotational flow, $u_{g}=x ; v_{g}=-y / 2$.

At the initial moment of time, the following conditions are set: $\delta(0)=1 ; x(0)=x_{0} ; y(0)=1 ; v_{m}(0)=-0.5$.

For irrotational flow, $u_{g}(0)=x_{0}$; for rotational flow, $u_{g}(0)=0$. The condition that the particle resides in the channel is defined by the following inequality: $x_{0}<x<l$, where $l$ is dimensionless length of channel.

To calculate the combustion efficiency of burning particles considering the velocity lag we use the following formula [16]:

$$
E=1-\frac{1}{l} \int_{0}^{1} \delta^{3}\left(x_{0}, l\right) d x_{0} .
$$

Here $\delta\left(x_{0}, l\right)$ is a diameter of particle at the exit area $(x=$ $l$ ) which left the burning propellant surface at the point with coordinate $x_{0}$. To provide $E=1$, it is necessary to resolve the system of (27) and to define a value of the $x_{0}$ coordinate which gives a value of $\delta\left(x_{0}, l\right)$ equal to zero.

From the above system of (27)-(30), one can see that the solution depends on, besides $\Phi$ and $\theta$, also three dimensionless parameters $l, \operatorname{Re}$, and $\Psi$, where $\Psi$ is the relaxation time to average residence time ratio. When $\Psi \rightarrow 0$, the flow tends to the equilibrium one.

As the propellant burns in the case of a cylindrical channel grain, the outflow velocity of the combustion products from the burning propellant surface changes. It is known [19] the combustion gas velocity at the burning surface is inversely proportional to the channel radius in this case, and the mass flux is proportional to the channel radius raised to the power of $n /(n-1)$, where $n$ is an exponent in the burning propellant rate law. Thus, dimensionless parameters $l, \theta, \Psi$, Re are also variable. If we denote these parameters at the initial moment of time as $l_{0}, \theta_{0}, \Psi_{0}, \operatorname{Re}_{0}$, then at another moment of time (in the case of $\left.A / S_{\mathrm{bs}} \sim 1 / R_{k}\right)$ they are as follows:

$$
\begin{gathered}
\psi=\frac{\psi_{0}}{\eta^{2}} ; \quad \theta=\frac{\theta_{0}}{\eta^{2}} ; \\
\operatorname{Re}=\operatorname{Re}_{0} \eta^{n /(n-1)} ; \quad l=\frac{l_{0}}{\eta} .
\end{gathered}
$$




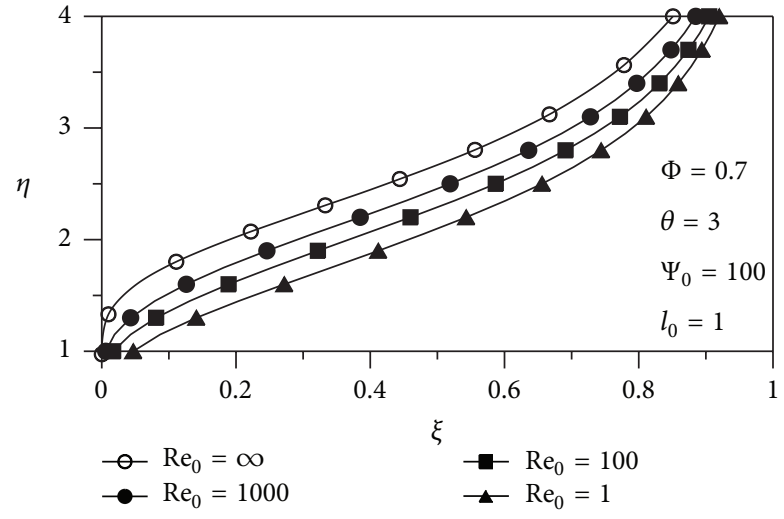

FIGURE 8: Effect of Re on optimum profile of metal content.

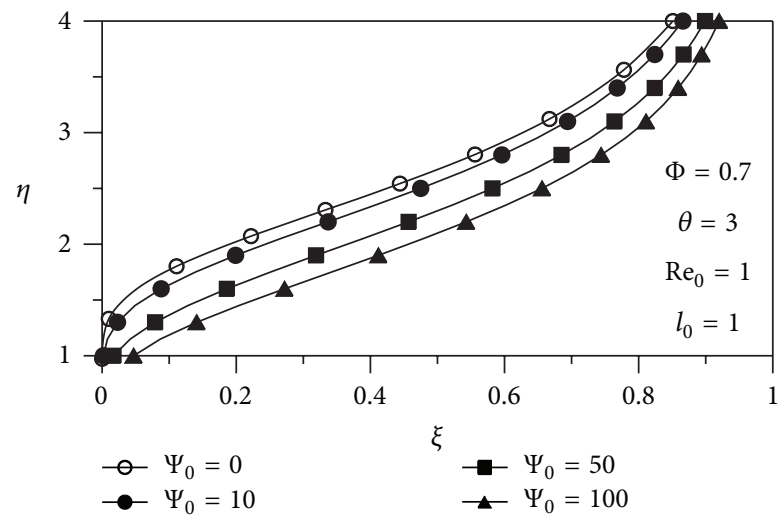

Figure 9: Effect of $\Psi_{0}$ on optimum profile of metal content.

Solving the system of (27)-(30), the dependent $\eta(\xi)$ which represents the optimum disposition of metal particles in the propellant can be obtained.

The influence of parameters $l_{0}, \Psi_{0}, \operatorname{Re}_{0}$ on the position of the curve $\eta(\xi)$ for rotational flow is shown in Figures 8, 9, and 10, respectively. The decrease of values of $l_{0}, \mathrm{Re}_{0}$ and the increase of value of $\Psi_{0}$ lead to the increase of the deviation of the curve position in the direction of the section $\xi=1$ from that corresponding to the equilibrium flow. As the shift of the curve $\eta(\xi)$ in that direction means the increase of the combustion efficiency, then the increase of the value of $\Psi_{0}$ and the decrease of the values of $l_{0}, \mathrm{Re}_{0}$ are preferable with the energetic point of view in the case of the nonequilibrium flow. It is clear that the closer the curve $\eta(\xi)$ passes near the curve corresponding to the equilibrium flow, the better the dependent (25) responds to the solution of the system of (27). Therefore, one can say that the dependent (25) describes the solution of the system (27) at large values of $l_{0}$ and $\operatorname{Re}_{0}$ and small $\Psi_{0}$.

\section{Conclusion}

The analytical correlation for the optimum disposition of metal particles in the case-bounded propellant grain of tube

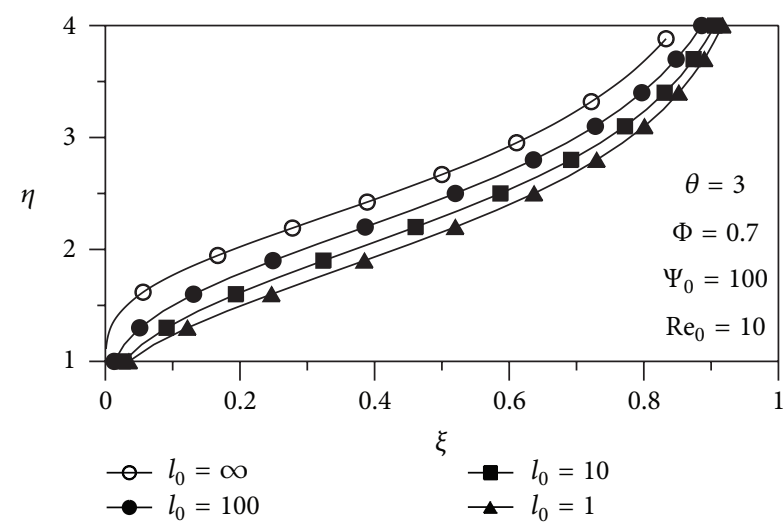

FIGURE 10: Effect of $l_{0}$ on optimum profile of metal content.

cross-sectional type under the assumption of equilibrium two-phase flow is deduced.

Analysis of the dimensionless system of equations describing a motion of burning particle in the channel of circular cross-section with taking into account the velocity lag shows that the optimum disposition of metal particles in the propellant grain depends on five parameters: the relative length of the channel; the particle relaxation time; the molar metal to oxidizer flux ratio at the propellant grain surface; particle combustion to residence time ratio; and Reynolds number of particle.

The increase of the particle relaxation time and the decrease of the particle Reynolds number and the relative channel length are preferable from the combustion efficiency point of view in the case of nonequilibrium flows.

\section{Nomenclature}

A: $\quad$ Nozzle throat area

D: $\quad$ Particle diameter

E: $\quad$ Metal combustion efficiency

$f, g, h$ : Functions

I: $\quad$ Inert gas

$\dot{G}: \quad$ Mass rate

$k$ : $\quad$ Coefficient in the particle burning rate law

$L: \quad$ Channel length

$l$ : Dimensionless channel length

M: $\quad$ Molar mass of species

$n$ : $\quad$ Exponent in the burning propellant rate law

$\mathrm{O}$ : $\quad$ Oxidizing gas

$p$ : $\quad$ Exponent in the particle burning rate law

$R: \quad$ Gas constant

$R_{k}: \quad$ Radius of channel

Re: Reynolds number

S: $\quad$ Lateral surface area of grain

$t: \quad$ Time

T: $\quad$ Temperature

$U, V: \quad$ Axial and radial components of velocity

$u, v: \quad$ Dimensionless components of velocity

$w$ : Velocity at the burning surface

$W$ : Volume 
$X, Y:$ Axial and radial coordinates

$x, y$ : Dimensionless coordinates

$z$ : $\quad$ Mass fraction of metal.

\section{Greek Symbols}

$\alpha$ : Molar fraction of oxidizer

$\beta$ : Volume fraction

$\delta$ : Dimensionless particle size

$\Gamma$ : Gas dynamic complex

$\gamma$ : Specific heat capacity ratio of gas phase

$\lambda$ : Drag function

$\rho$ : Bulk density

$\tau$ : Dimensionless time

$\mu$ : Viscosity

$\nu$ : Stoichiometric coefficient

$\theta$ : Burning-to-residence time ratio

$\Phi$ : Mass flux ratio

$\omega$ : Molar mass complex

$\Psi$ : Relaxation-to-residence time ratio.

\section{Subscripts}

$\begin{array}{ll}c: & \text { Oxide } \\ c c: & \text { Combustion chamber } \\ b: & \text { Burning } \\ \text { bi: } & \text { Binder } \\ \text { bs: } & \text { Burning surface } \\ i: & \text { Inert gas } \\ g: & \text { Gas phase } \\ \text { lim: Limit } \\ m: \text { Metal } \\ \text { mb: Metal in binder } \\ o: \text { Oxidizing gas } \\ \text { ox: Oxidizer particle } \\ \text { out: Channel outlet } \\ p: \text { Combustion products } \\ r: & \text { Residence } \\ 0: & \text { Initial value. }\end{array}$

\section{Conflict of Interests}

The authors declare that there is no conflict of interests regarding the publication of this paper.

\section{Acknowledgment}

Support for this work was provided by the Ministry of Education and Science of Russian Federation, Agreement no. 10.1329.2014/K.

\section{References}

[1] F. Maggi, G. Gariania, L. Galfettia, and L. T. de Luca, "Theoretical analysis of hydrides in solid and hybrid rocket propulsion," International Journal of Hydrogen Energy, vol. 37, no. 2, pp. 17601769, 2012.
[2] D. R. Greatrix, "Multisized inert particle loading for solid rocket axial combustion instability suppression," International Journal of Aerospace Engineering, vol. 2012, Article ID 173129, 13 pages, 2012.

[3] R. A. Yetter, G. A. Risha, and S. F. Son, "Metal particle combustion and nanotechnology," Proceedings of the Combustion Institute, vol. 32, pp. 1819-1838, 2009.

[4] F. Maggi, A. Bandera, L. Galfetti, L. T. De Luca, and T. L. Jackson, "Efficient solid rocket propulsion for access to space," Acta Astronautica, vol. 66, no. 11-12, pp. 1563-1573, 2010.

[5] F. Daidzo, N. Tadahiko, K. Tekamega, and S. Sedzo, "Composition of the Propellant," Journal of Abstracts, no. 14 (III), 1978 (Russian).

[6] V. N. Vilyunov, A. B. Vorozhtsov, and Y. V. Feshchenko, "Modeling of two-phase flow of a gas mixture with burning metal particles in a semienclosed channel," Combustion, Explosion and Shock Waves, vol. 25, no. 3, pp. 296-300, 1989.

[7] E. D. Terzi, "Analytical approach to the problem of incomplete combustion of metal additives in the reactors," in Mechanics of Fast Processes, pp. 50-57, Tomsk State University, Tomsk, Russia, 1989, (Russian).

[8] F. Maggi, S. Dossi, and L. T. de Luca, "Combustion of metal agglomerates in a solid rocket core flow," Acta Astronautica, vol. 92, no. 2, pp. 163-171, 2013.

[9] K. N. Volkov, "Motion of a condensed particle in a channel with injection," Journal of Engineering Physics and Thermophysics, vol. 79, no. 1, pp. 83-92, 2006.

[10] K. N. Volkov, "Application of the parameter-expansion method to calculation of two-phase flows in channels with injection," Journal of Engineering Physics and Thermophysics, vol. 79, no. 6, pp. 1169-1178, 2006.

[11] K. N. Volkov and B. H. Emelyanov, Particle Laden Flows, Fizmatlit, Moscow, Russia, 2008 (Russian).

[12] V. D. Lisitza, "Influence of mass forces on the radial structure of two-phase flow in the channel of circular cross-section," Polzunovskii Vestnik, no. 1-2, pp. 85-91, 2008 (Russian).

[13] K. N. Volkov, "Qualitative analysis and numerical simulation of the movement of a particle in a channel having permeable walls with account for the action of mass forces," Journal of Engineering Physics and Thermophysics, vol. 86, no. 6, pp. 12861293, 2013.

[14] M. W. Beckstead, "Correlating aluminum burning times," Combustion, Explosion and Shock Waves, vol. 41, no. 5, pp. 533-546, 2005.

[15] A. F. Belyaev, Y. V. Frolov, and A. I. Korotkov, "Combustion and ignition of particles of finely dispersed aluminum," Combustion, Explosion, and Shock Waves, vol. 4, no. 3, pp. 182-185, 1971.

[16] R. S. Larson, "Prediction of aluminum combustion efficiency in solid propellant rocket motors," AIAA journal, vol. 25, no. 1, pp. 82-91, 1987.

[17] S. W. Jane and A. B. Finkelstein, "Laminar pipe flow with injection and suction through a porous wall," Transactions of ASME, vol. 78, no. 4, pp. 719-724, 1956.

[18] L. G. Loitsyanskii, Mechanics of Liquids and Gases, Pergamon Press, New York, NY, USA, 1966.

[19] B. T. Erokhin, Theory of Intrachamber Processes and Design of SRM, Mashinostroenie, Moscow, Russia, 1991 (Russian). 

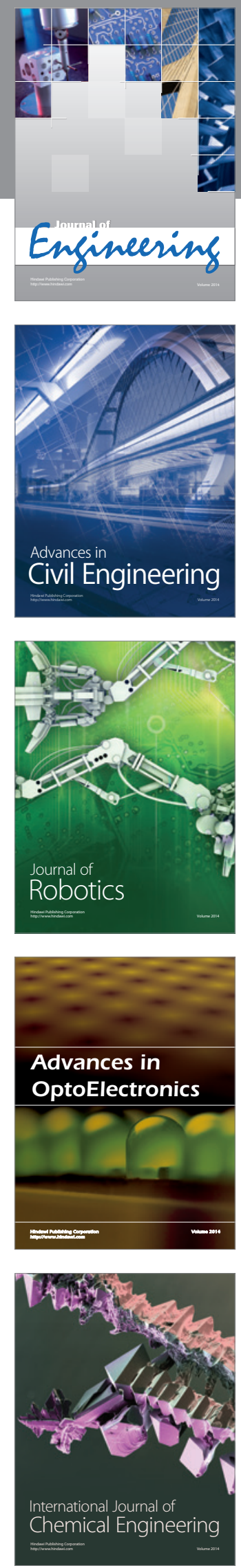

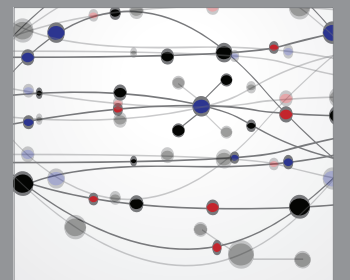

The Scientific World Journal
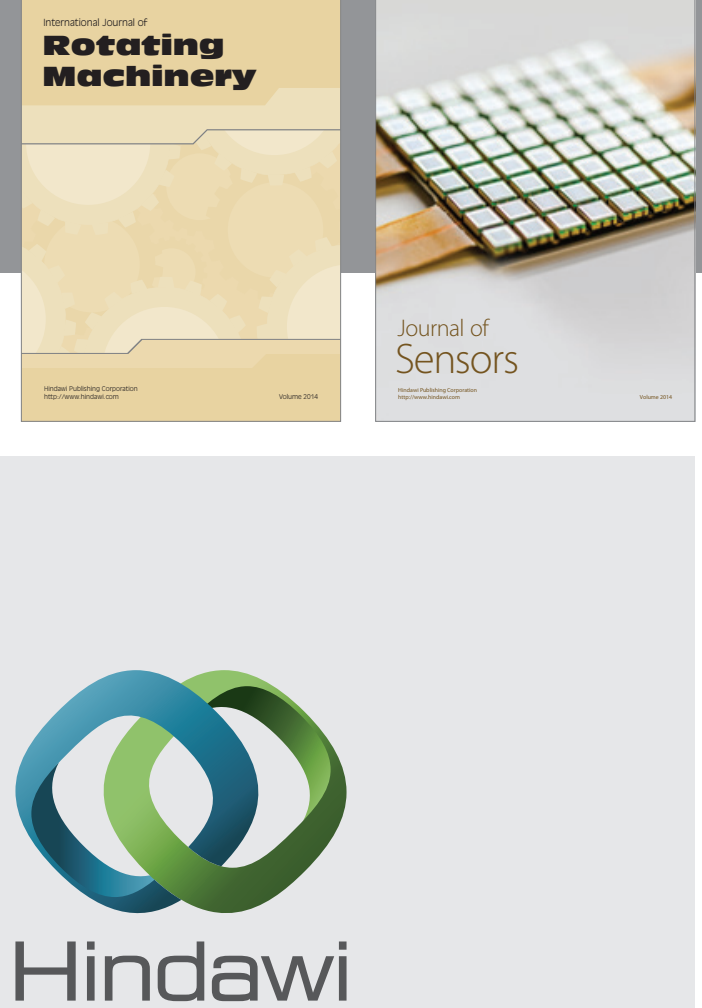

Submit your manuscripts at http://www.hindawi.com
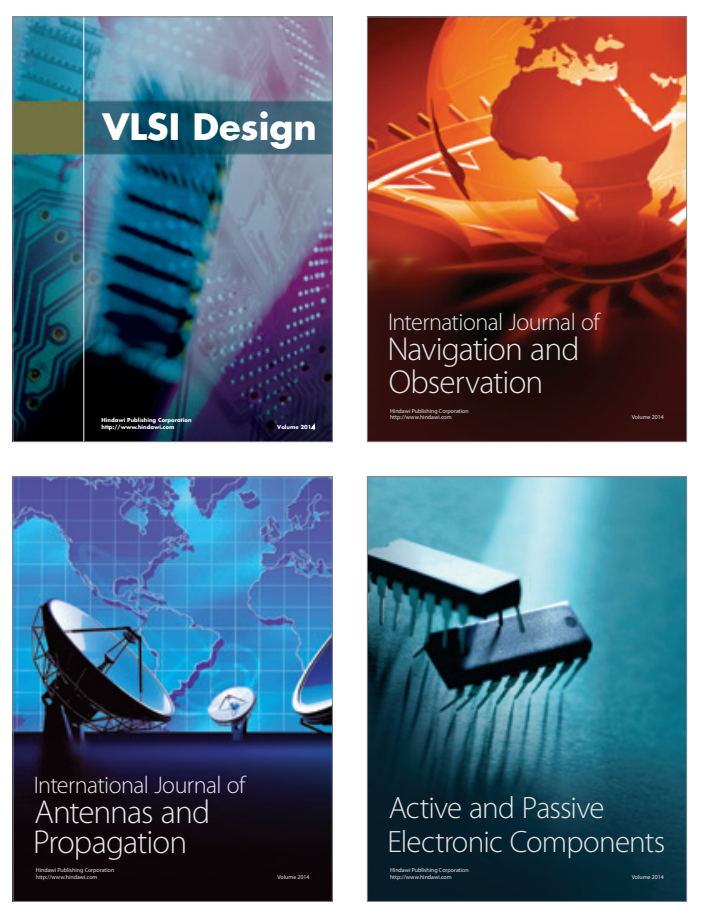
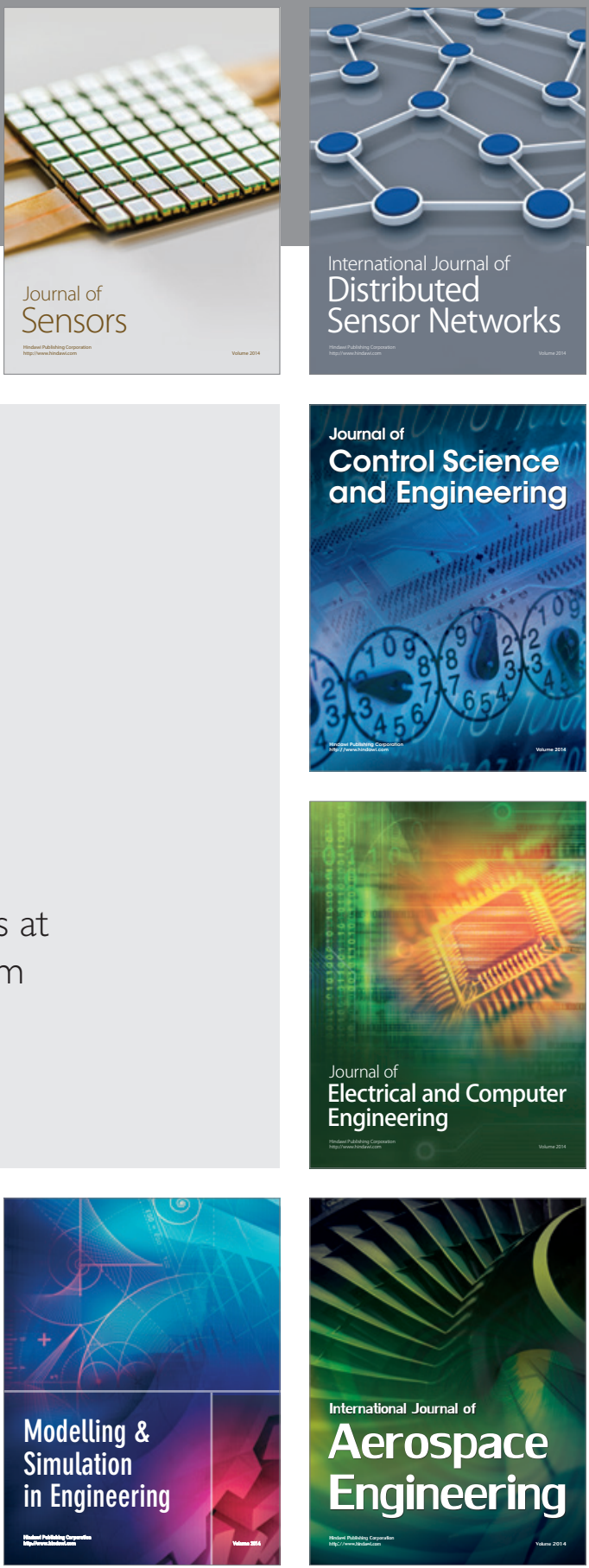

Journal of

Control Science

and Engineering
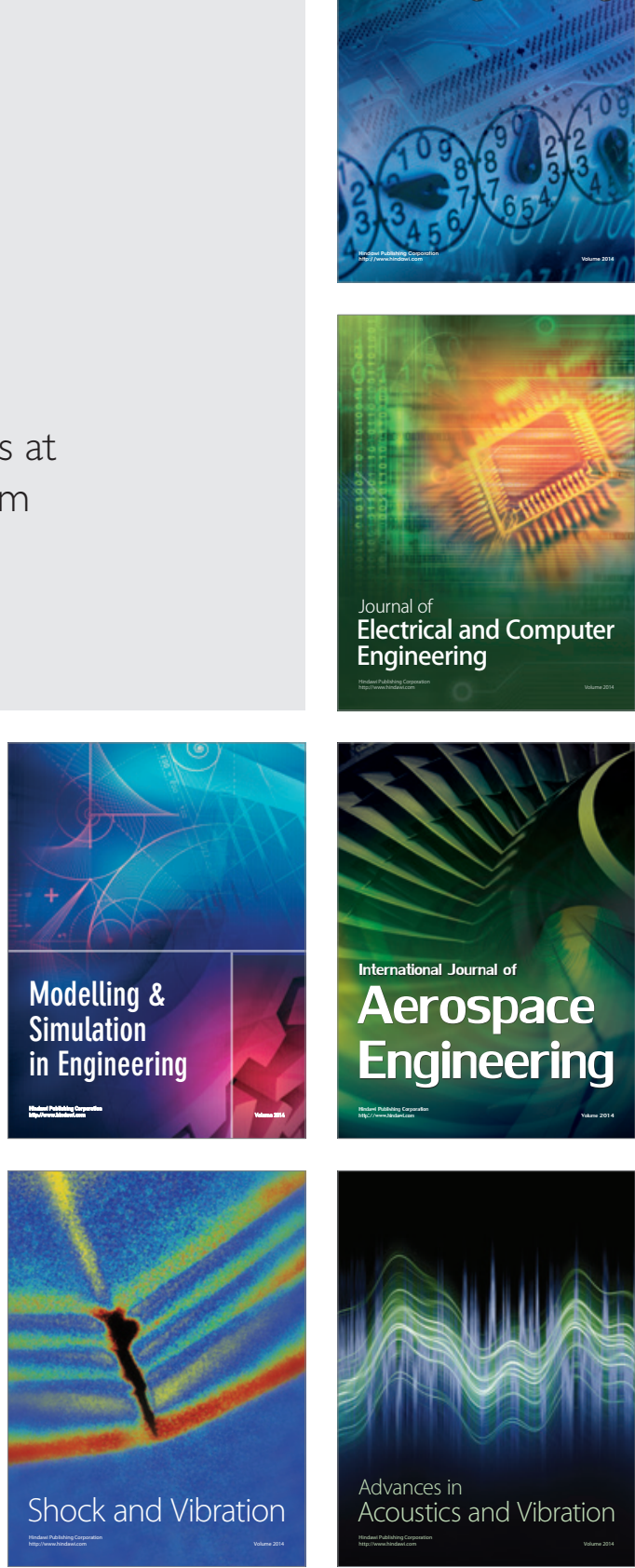